\title{
Short-Term GDNF Treatment Provides Long-Term Rescue of Lesioned Nigral Dopaminergic Neurons in a Rat Model of Parkinson's Disease
}

\author{
Christian Winkler, ${ }^{1,2}$ Hansjörg Sauer, ${ }^{1,3}$ Chong S. Lee, ${ }^{1,4}$ and Anders Björklund ${ }^{1}$ \\ 1Lund University, Wallenberg Neuroscience Center, S-22362 Lund, Sweden, ${ }^{2}$ Neurosurgical Clinic, Nordstadt Hospital, \\ D-30167 Hannover, Germany, ${ }^{3}$ Genentech Inc., South San Francisco, California 94080, ${ }^{4}$ Neurodegenerative Disorders \\ Centre, Vancouver Hospital and Health Sciences Centre, Vancouver, British Columbia, Canada V6T 2B5
}

Glial cell line-derived neurotrophic factor (GDNF) has been shown to exert neuroprotective effects on dopamine (DA) neurons in vivo. Here we report long-term rescue of nigral DA neurons after delayed short-term GDNF administration in a rat lesion model that reproduces the slowly progressing degenerative process seen in Parkinson's disease. GDNF injected close to the substantia nigra provided near-complete protection and persistent survival of the lesioned nigral neurons for at least 4 months after discontinuation of GDNF treatment. Long-term rescue of the nigral cells, however, was not accompanied by any significant reinnervation of the lesioned striatal target or any signs of functional recovery in either drug-induced or spontaneous motor behaviors. We conclude that not only preservation of the nigral DA neurons but also restoration of striatal DA function is necessary for functional recovery in the rat Parkinson model.

Key words: GDNF; substantia nigra; striatum; neurodegeneration; behavior; Parkinson's disease
Parkinson's disease (PD) is a neurodegenerative disorder characterized by progressive degeneration of the dopamine (DA) neurons in the substantia nigra $(\mathrm{SN})$ that develops over years and decades. Symptoms occur when $\sim 80 \%$ of striatal DA and $50 \%$ of the nigral cells are lost (Riederer and Wuketich, 1976). The severity of the disease has been shown to be correlated with DA neuronal cell loss in the SN (Mc Geer et al., 1988; Fearnley and Lees, 1991), and there is a progressive reduction in striatal $\left[{ }^{18} \mathrm{~F}\right]$ fluorodopa uptake, which is consistent with the view that the neurodegenerative process progresses over many years before any symptoms appear (Sawle and Myers, 1993). The progressive nature of the disease suggests interesting possibilities for therapeutic intervention by blocking the underlying neurodegenerative process. The search for neurotrophic factors with potent and specific actions on DA neuron survival is therefore of considerable interest.

Glial cell line-derived neurotrophic factor (GDNF) has been shown to possess potent neurotrophic effects on DA neurons in vitro (Lin et al., 1993) and to exert neuroprotective effects in vivo. GDNF has been shown to rescue nigral DA neurons from lesioninduced cell death after surgical- or toxin-induced axotomy in rats (Beck et al., 1995; Kearns and Gash, 1995; Sauer et al., 1995) and partially also after systemic administration of $N$-methyl-4-phenyl1,2,3,6-tetrahydropyridine (MPTP) in mice (Tomac et al., 1995). In these studies, the neuroprotective effect of GDNF was ob-

Received May 22, 1996; revised Aug. 19, 1996; accepted Aug. 22, 1996.

This study was supported by grants from the Swedish MRC (04X-3874) and the Göran Gustafsson Foundation. C.W. was supported by a fellowship from the European Neuroscience Program of the European Science Foundation. We thank Drs. A. Rosenthal and K. Poulsen, Genentech Inc., for the generous supply of GDNF for these experiments, and Cristina Ciornei, Sten Nilsson, and Gertrude Stridsberg for expert technical support and animal care. C.W. thanks Prof. Madjid Samii and Dr. Guido Nikkhah for generous support.

Correspondence should be addressed to Dr. Christian Winkler, Lund University, Wallenberg Neuroscience Center, Sölvegatan 17, S-22362 Lund, Sweden.

Copyright (C) 1996 Society for Neuroscience $0270-6474 / 96 / 167206-10 \$ 05.00 / 0$ served after local injection into the mesencephalon, close to the $\mathrm{SN}$, when administered either before or immediately after the insult. In addition, Bowenkamp et al. (1995) and Gash et al. (1996) have reported that single high doses of GDNF, injected 1 or 3 months after the toxic insult, can induce upregulation of tyrosine hydroxylase $(\mathrm{TH})$ expression in the injured nigral DA neurons.

Neurotoxic lesions of striatal DA nerve terminals, induced by intrastriatal 6-OHDA administration, are particularly interesting as an experimental model for the study of neuroprotective agents, because the retrograde degenerative changes induced in the damaged SN neurons progress slowly over several weeks, resulting in a dose-dependent cell loss and atrophy of the surviving DA neurons (Sauer and Oertel, 1994; Przedborski et al., 1995; Lee et al., 1996). The behavioral impairments, as observed in tests of both drug-induced turning and spontaneous motor behaviors, have been shown to correlate well with the magnitude of cell loss in the SN (Przedborski et al., 1995; Lee et al., 1996). This partial lesion model thus leaves a time window of several weeks to investigate the effects of neuroprotective agents on both DA neuron atrophy and cell death as well as functional recovery.

Previous reports on the in vivo neuroprotective effects of GDNF in the nigrostriatal system have been limited to studies on the short-term actions of the factor 1-4 weeks after the neurotoxic or surgical insult. Thus, it is not yet known whether the GDNFinduced neuroprotective effects are long-lasting and to what extent rescue of nigral DA neurons by administration of GDNF over the SN can afford significant preservation of motoric behaviors of relevance for the symptomatology of human PD. The present study, therefore, was designed to examine the long-term neuroprotective and regenerative effects of delayed, short-term GDNF treatment and to investigate the effects of intranigral GDNF administration on the behavioral deficits in the partial lesion model, as measured by a battery of motor initiation and akinesia tests. 


\section{MATERIALS AND METHODS}

Animals and surgery. Young adult female Sprague Dawley rats (B \& K Universal, Stockholm, Sweden), weighing 200-250 gm at the beginning of the experiment, were housed under a $12 \mathrm{hr}$ light/dark cycle with access to food and water ad libitum. Two stereotaxic injections of 6-OHDA were made under equithesin anesthesia into the right striatum using a $10 \mu \mathrm{l}$ Hamilton microsyringe fitted with a 26 gauge steel cannula. A total amount of 6-OHDA (20 $\mu \mathrm{g})$ dissolved in $4 \mu \mathrm{l}$ of $0.2 \mathrm{mg} / \mathrm{ml} \mathrm{L}$-ascorbatesaline (10 $\mu \mathrm{g}$ in $2 \mu \mathrm{l}$ per site) was injected at the following coordinates (in $\mathrm{mm}$, with reference to bregma and dura): (1) anterior-posterior, 0.5; lateral, -2.5 ; and ventral, -5.0 [tooth bar (TB), 0] and (2) anteriorposterior, -0.5 ; lateral, -4.2 ; and ventral, -5.0 (TB, 0$)$. The injection rate was $1 \mu \mathrm{l} / \mathrm{min}$, and the cannula was left in place for additional $2 \mathrm{~min}$ before slowly retracting it.

During the same surgery session, animals received implants of 22 gauge guide cannulae (Plastics One, Roanoke, VA) over the SN on the lesioned side. Coordinates for guide cannula implantation were anterior-posterior, -5.2; lateral, $2.0(\mathrm{~TB},-3.3)$, and cannulae were shortened so as to penetrate $1-1.5 \mathrm{~mm}$ into the neocortex. The guide cannulae were anchored to the skull with three jeweler screws and dental cement and were sealed with 26 gauge dummy cannulae after surgery. Animals were left to recover for $4 \mathrm{~d}$ after lesion surgery and implantation of guide cannulae.

Beginning on day 5 after lesion surgery, animals received injections of $10 \mu \mathrm{g} \mathrm{GDNF} / 1 \mu \mathrm{l}$ saline (the kind gift of A. Rosenthal and K. Poulsen, Genentech), or saline alone, through the guide cannulae into the midbrain, $1-1.5 \mathrm{~mm}$ dorsal to the $\mathrm{SN}$ on the lesioned side, using a 28 gauge injection cannula connected to a $10 \mu \mathrm{l}$ Hamilton syringe with a piece of plastic tubing. Injections of GDNF or saline were made under light halothane sedation every fourth day. After 9 GDNF or saline injections, i.e., at $37 \mathrm{~d}$ after lesion surgery and $32 \mathrm{~d}$ after the first intracerebral GDNF or saline injection, treatment was discontinued and animals were left alive for another 4 months, during which time they were subjected to a range of tests of sensorimotor behaviors.

Rotational behavior. At $4 \mathrm{~d}, 11 \mathrm{~d}$, and 4 weeks after lesion surgery, amphetamine-induced rotational behavior was measured in automated rotometer bowls according to Ungerstedt and Arbuthnott (1970), with $4 \mathrm{~d}$ representing the time point before the first GDNF or vehicle injection. The animals were given $5 \mathrm{mg} / \mathrm{kg}$ D-amphetamine intraperitoneally, and left and right full-body turns were monitored over a 90 min period. Net rotational asymmetry score is expressed as full-body turns per minute in the direction ipsilateral to the lesion.

Stepping test. At 4 months after lesioning, forelimb akinesia was examined in a side-stepping test, as described by Olsson et al. (1995). On the $2 \mathrm{~d}$ before the actual test, the rats were handled by the experimenter to become familiar with the grip used during testing. During the subsequent $3 \mathrm{~d}$ of testing, the initiation of adjusting steps was monitored when the animals were passively moved sideward along a table surface. Briefly, the rat was held by a "blind" experimenter fixing its hindlimbs with one hand and the forelimb not to be monitored with the other while the unrestrained forepaw was touching the table. The number of adjusting steps was counted while the rat was slowly moved sideways along the table surface $(0.9 \mathrm{~m}$ in $5 \mathrm{sec})$, first in the forehand and then in the backhand direction. The test was performed for each forelimb in both movement directions. It was repeated twice a day on three consecutive days, and the average of the six subtests was calculated.

Balance test. In the same test sessions, the rats' ability to regain balance was measured in a balance or side-falling test. The rat was held in the same position as described in the stepping test, with the rat standing with one forepaw on the table. Instead of being moved sideways, the rat was tilted by the experimenter toward the side of the paw touching the table. This resulted in loss of balance, and the ability of the rat to regain balance by movement of the forelimb during the tilting movement was monitored by a scoring system ranging from 0 to 3 . When the rat fell onto the side and there was no detectable muscle reaction in the forelimb, score 0 was given. Score 1 represented a clear forelimb reaction, as seen by muscle contractions, but lack of success in recovering balance, i.e., the rat still fell onto the side. Score 2 was given when the rat showed an incomplete recovery of balance, i.e., the rat performed a clear forelimb movement, but the placement of the paw was impaired compared with that seen in controls in that the digits were not plainly spread on the table but partly crossed over one another. Score 3 was given for a normal forelimb placement movement and total recovery of balance, similar to unlesioned controls. The test was repeated six times a day on each side for three consecutive days, giving a maximal score of 18 at each of the three test days. Final results are expressed as the mean of the $3 \mathrm{~d}$.
Sensorimotor orientation and disengage behavior. At 4.5 months after lesioning, a test of sensorimotor orientation and disengage behavior was performed by a naive observer. According to the protocol of Schallert and Hall (1988) and Mandel et al. (1990), the perioral region beneath the vibrissae was touched repeatedly by a wooden probe at $1 \mathrm{sec}$ intervals until an orientating response of the rat toward the wooden probe was recorded. The time to initiation of a response was recorded. An immediate response was scored as $1 \mathrm{sec}$. Sensorimotor orientation was examined when the animals were sitting absolutely still in their cage, whereas disengage behavior was recorded when the animals were engaged in eating a piece of milk chocolate. The animals were tested twice daily on each side for two consecutive days.

TH immunohistochemistry. At 5 months after lesioning, the animals were deeply anesthetized with chloral hydrate and perfused through the ascending aorta with $30 \mathrm{ml}$ of $0.9 \%$ saline, followed by $300 \mathrm{ml}$ of ice-cold $4 \%$ paraformaldehyde in $0.1 \mathrm{M}$ phosphate buffer (PB), $\mathrm{pH}$ 7.4. After post-fixation for $2 \mathrm{hr}$ in PFA and overnight dehydration in 20\% sucrose/ $0.1 \mathrm{M} \mathrm{PB}$, coronal sections were cut at $30 \mu \mathrm{m}$ thickness on a freezing microtome, and every third section was processed for free-floating $\mathrm{TH}$ immunohistochemistry. The sections were rinsed three times in $\mathrm{PB}$, quenched for $10 \mathrm{~min}$ in $3 \% \mathrm{H}_{2} \mathrm{O}_{2} / 10 \%$ methanol/PB, and rinsed in $\mathrm{PB}$. After a $1 \mathrm{hr}$ preincubation in $5 \%$ normal swine serum (NSS)/0.3\% Triton $\mathrm{X}-100 / \mathrm{PB}$, sections were incubated overnight in a 1:500 dilution of primary anti-rat TH antiserum (Pel Freez) in 2\% NSS/0.3\% Triton X-100/ PB. After three rinses with PB, sections were incubated in a 1:200 dilution of biotinylated swine anti-rabbit IgG (Dakopatts)/0.3\% Triton X-100/PB for $1 \mathrm{hr}$, rinsed three times, and transferred to a Vectastain $\mathrm{ABC}$ solution/PB for $1 \mathrm{hr}$. 3,3-Diaminobenzidine (0.05\%) (Sigma, St. Louis, $\mathrm{MO}$ ) in $0.01 \% \mathrm{H}_{2} \mathrm{O}_{2} / \mathrm{PB}$ served as chromogen in the subsequent visualization reaction. Sections were mounted on chromalum-coated slides, dehydrated in ascending alcohol concentrations, cleared in xylene, and coverslipped in DPX.

Microscopic analysis. For quantitative analysis of number and size of TH-immunoreactive (TH-IR) neurons in the SN, three sections per series were selected from each animal that showed a clear separation of SN and ventral tegmental area (VTA) by the medial terminal nucleus of the accessory optic tract (MTN) level anterior-posterior $-5.3 \mathrm{~mm}$ from bregma in the atlas of Paxinos and Watson (1986). This landmark, which could be seen in three sections per series, helped to define in a reproducible way the midportion of the SN. The lesion-induced cell loss in this part has been shown to be representative for the entire nucleus (Sauer and Oertel, 1994; Sauer et al., 1995).

TH-IR neurons in the SN were counted under $20 \times$ objective magnification in coded sections on the lesioned and contralateral sides under bright-field illumination. Cell numbers are expressed either as the mean number per section from these three sections or as percent of the contralateral side.

Cell size was measured as the cross-sectional area (in $\mu \mathrm{m}^{2}$ ) covered by the TH-IR cell profiles using a computer-based image analysis system (Image 1.55, Wayne Rasband, National Institute of Mental Health, Bethesda, MD) run on a Macintosh Centris 650. A minimum of 80 TH-IR cells on each side, or all surviving cells in animals with severe lesioninduced cell loss, was measured in the pars compacta of the SN in the same coded sections selected for assessment of cell numbers.

Striatal TH-IR fiber density was determined with the same image analysis system used for the assessment of cell size. The whole crosssectional area of the striatum was outlined on the computer screen, and the fiber density was measured as mean optical density expressed in percent of the density recorded from the same area of the striatum on the contralateral side. As background value, the mean optical density of the corpus callosum was recorded and subtracted from the striatal optical density to give a final striatal optical density. Fiber density was determined at three rostrocaudal levels: (1) rostral striatum [anterior-posterior, $+1.3 \mathrm{~mm}$ in the atlas of Paxinos and Watson (1986)]; (2) lesion site, recorded as the mean of three sections, two at the level of each of the two 6-OHDA lesions (anterior-posterior, $+0.5 \mathrm{~mm}$ and $-0.5 \mathrm{~mm}$, and one in between these two levels); (3) caudal striatum (anterior-posterior, -1.1 $\mathrm{mm}$ ). In addition, TH-IR fiber density was determined in the ventrolateral part of the striatum at AP level $+1.0 \mathrm{~mm}$.

Statistical analysis. For intergroup comparisons, data were subjected to a one-factor ANOVA, followed by a post hoc Scheffé test. Comparisons between lesioned and nonlesioned sides within the the same group were performed using paired Student's $t$ test. Linear regression analysis was used to assess possible correlations between different morphometric and 
behavioral measures. Statistical significance level was set at $p<0.05$ for all tests.

\section{RESULTS}

Transient GDNF treatment protects nigral DAergic cells from lesion-induced cell death

6-OHDA was injected as two $10 \mu \mathrm{g}$ deposits in the centrolateral part of the caudate-putamen. Starting $5 \mathrm{~d}$ after the neurotoxin lesioning, GDNF was injected through an indwelling guide cannula into the midbrain every fourth day for 1 month $(10 \mu \mathrm{g}$ in 1 $\mu \mathrm{l} / \mathrm{d}$, nine injections in total). Controls received similar injections of the saline vehicle. Microscopic analysis of the injection sites showed that the injection was located $\sim 1 \mathrm{~mm}$ dorsal to the $\mathrm{SN}$ pars compacta. Tissue damage at the injection site was small, and neither necrosis nor extensive vacuolization was observed in the surrounding host midbrain tissue in either the GDNF- or vehicleinjected animals.

At 5 months after the intrastriatal 6-OHDA lesioning, and 4 months after the last GDNF or vehicle injection, TH-IR cell counts in the SN on the lesioned side were reduced to $19.4 \pm 4 \%$ of the contralateral intact side in animals that had received vehicle injections over the $\mathrm{SN}(p<0.001)$ (Figs. $1 D, 2)$. In contrast, there was significant sparing of the nigral TH-IR neurons, by $77.8 \pm$ $4.1 \%$ of contralateral side, in the GDNF-injected animals $(p<$ 0.001) (Figs. $1 F, 2$ ). As shown by Sauer and Oertel (1994), reduced numbers of TH-IR neurons in this paradigm reflect cell death and not downregulation of the enzyme. In all GDNFtreated animals, but not in the vehicle-injected animals, there was in addition clear sparing of the TH-IR fibers in the SN pars reticulata with close to normal density of TH-IR dendritic fibers, similar to that seen on the contralateral intact side. In the GDNFtreated animals, but not in the vehicle group, there were clear signs of sprouting of TH-IR fibers from the cell bodies in the SN pars compacta toward the injection site (Fig. $1 F$ ).

In both the vehicle- and GDNF-treated animals, the surviving TH-IR neurons were significantly reduced in size, from $182.5 \pm$ $1.5 \mu \mathrm{m}^{2}$ (cross-sectional area per cell) on the nonlesioned side versus $152.4 \pm 3.8 \mu \mathrm{m}^{2}$ on the lesioned side in the vehicle group $(p<0.001)$ (Fig. 2) and from $184.9 \pm 1.4 \mu \mathrm{m}^{2}$ on the nonlesioned side to $152.0 \pm 3.4 \mu \mathrm{m}^{2}$ on the lesioned side in the GDNF group $(p<0.001)$ (Fig. 2). This signifies that the TH-positive neurons that were rescued by the transient GDNF treatment remained in an atrophic state.

\section{GDNF administered over the cell bodies fails to protect the DAergic nerve terminals in the striatum}

The intrastriatal 6-OHDA lesion caused a variable loss of TH-IR fibers in the striatum, with some specimens displaying an almost complete loss of TH-IR in a large part of the centrolateral portion of the caudate-putamen (Fig. $3 B, E, H$ ), whereas other animals had smaller lesions confined to the dorsolateral part (Fig. $3 C, F, I)$. The quantitative densitometric measurements, summarized in Figure 4, showed that the 6-OHDA-induced axon terminal degeneration was most profound in the lateral and caudal part of the nucleus, close to the 6-OHDA injection sites, where $>90 \%$ of the TH-IR innervation was lost in the most extensively denervated specimens. Clearly more TH-IR fibers (on average $~ 50 \%$ ) were spared rostrally to the lesion (Fig. 4). Significant sparing occurred also in the ventrolateral striatum, in which the average TH-IR fiber density amounted to 56 and $63 \%$ of contralateral in the vehicle- and the GDNF-injected groups, respectively (Fig. 4).

There was clearly no protective effect of the GDNF treatment on the striatal DA nerve terminals. No differences in TH-IR striatal fiber density, measured as mean density in percent of the contralateral side at three rostrocaudal levels, were observed between the GDNF- and vehicle-injected animals $(p>0.4)$ (Fig. $4)$, and there was no indication of any TH-IR fiber sprouting within the striatum in the GDNF-treated group. The absolute values of TH-IR fiber density on the side contralateral to the lesion were not different in the two groups $(p>0.5)$, excluding a possible bilateral effect of the GDNF treatment.

\section{Lesion-induced behavioral impairments Amphetamine-induced rotation}

As illustrated in Figure $5 A$, amphetamine-induced ipsilateral rotation scores increased from 11.8 full-body turns per minute at $4 \mathrm{~d}$ to 18.8 turns per minute at 4 weeks after lesioning in the vehicleinjected group and from 17.2 to 18.1 turns per minute in the GDNF-injected group. Intergroup differences were not statistically significant.

\section{Stepping test}

The ability of the rats to initiate stepping movements was used as a measure of forelimb akinesia in the lesioned animals. As assessed at 4 months after surgery, the striatal 6-OHDA lesion induced marked impairments in the performance of the contralateral (left) paw, whereas the ipsilateral (right) paw was unaffected in both the forehand and the backhand direction (Fig. $5 B$ ). The left forelimb akinesia was unaffected by the GDNF treatment, and no differences in performance between the GDNF and vehicle groups were noted. As illustrated in Figure $5 B$, the lesion-induced effect was most pronounced in the forehand direction, with the adjusting steps being reduced by $>75 \%$ as compared with the ipsilateral paw ( $p<0.001$ for each experimental group). Although induction of stepping in the backhand direction was reduced by only $\sim 20 \%$ this effect was nevertheless statistically significant in both groups $(p<0.05)$. As discussed below, performance was not homogenous in the experimental groups. Complete absence of stepping in the forehand direction was thus observed in some animals, whereas others performed fairly well in the test (see Fig. 7B).

\section{Balance test}

In the balance test, the initiation of adjustment steps to regain balance was tested for each forelimb separately. Right paw performance was unaffected by the intrastriatal 6-OHDA lesion, and all animals achieved a balance test score corresponding to the behavior of nonlesioned animals (Fig. 5C) (maximum score, 18). On the left side, contralateral to the lesion, a similar pattern of impairment was observed in both the GDNF- and vehicle-injected groups (Fig. $5 C$ ). The balance test score on the left was reduced by $32 \%$ in the vehicle-injected group $(p<0.01)$ and by $40 \%$ in the GDNF-injected group $(p<0.02)$. Similar to the stepping test, the performance of the animals was quite variable within both groups, with single animals reaching normal balance test scores, whereas others were severely impaired (see Fig. 7C).

\section{Sensorimotor orientation and disengage behavior}

Sensorimotor orientation and disengage behavior are motor initiation tests that are known to be sensitive to extensive DA denervation of the ventrolateral sector of the striatal complex (Mandel et al., 1990). No lesion-induced impairment was observed in either the sensorimotor or the disengage behavior tests (data not shown); in both tests, all animals reacted immediately 

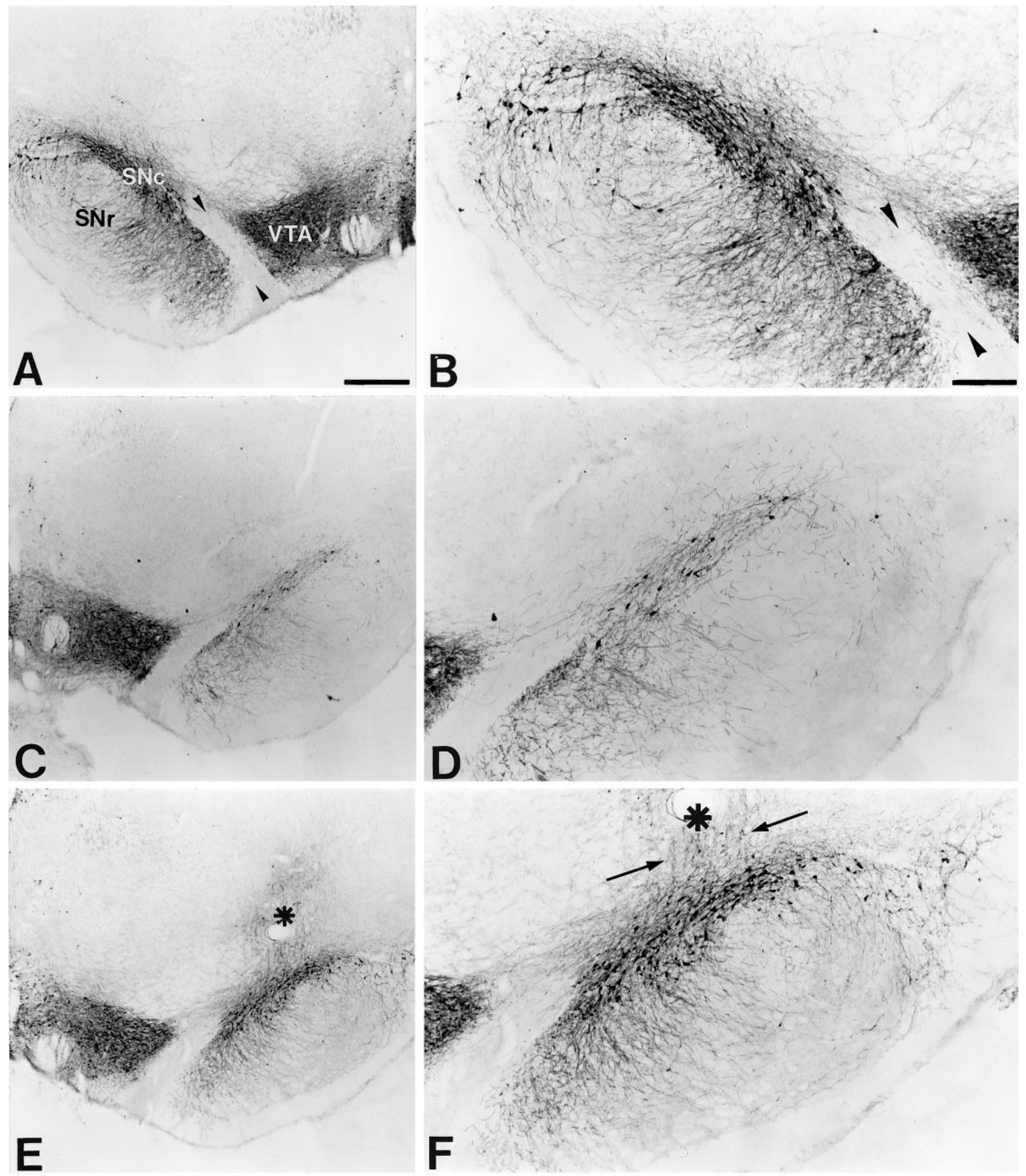

Figure 1. Transient GDNF treatment protects nigral DA neurons from lesion-induced cell death. Coronal sections through the SN and the VTA separated by the MTN (arrowheads). A, B, The side contralateral to the intrastriatal 6-OHDA lesion. Most of the TH-IR cells are located in the SN pars compacta $(S N c)$, whereas a few TH-IR cells and TH-IR fibers can be seen in the SN pars reticulata $(S N r)$. $C, D$, The midbrain ipsilateral to the 6-OHDA lesion in a vehicle-injected animal. Few TH-IR cells remain in the SN, and there is a marked loss of TH-IR fibers in the SNr. No obvious effect of the intrastriatal 6-OHDA lesion can be seen on TH-IR cells in the VTA. E, $F$, The side ipsilateral to the 6-OHDA lesion in a representative animal that had received transient GDNF treatment. Many TH-IR cell profiles can be seen in the SNc, and the TH-IR fiber density in the SNr is preserved at normal levels. The injection site is located dorsal to the SNc (asterisk), and apparent sprouting of TH-positive fibers from the SN toward the injection site can be seen (arrows). Scale bars: $A, 500 \mu \mathrm{m} ; B, 250 \mu \mathrm{m}$. 


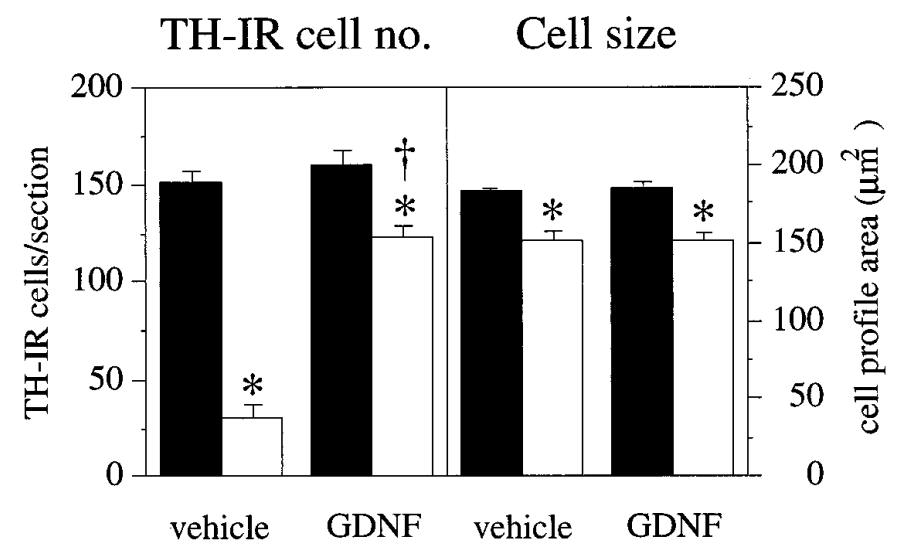

contralateral $\square$ ipsilateral to lesion

Figure 2. Nigral DA neurons are rescued by transient GDNF treatment but remain in an atrophic state. TH-IR cells in the SN were counted in sections that showed a clear separation of SN and VTA by the MTN (see Fig. 1). This landmark was seen in three sections per series. Five months after the intrastriatal 6-OHDA lesion and 4 months after cessation of either vehicle or GDNF injections, the number of TH-IR cells in the ipsilateral SN was reduced to $19.4 \pm 4 \%$ compared with the contralateral side in vehicle-injected animals, whereas $77.8 \pm 4.1 \%$ of the cells were spared in GDNF-injected animals. Left panel, Asterisks indicate significant difference from the contralateral side $(p<0.001$, paired $t$ test); dagger, significant difference from vehicle-injected group $(p<0.001$, ANOVA with post hoc Scheffé test). In both groups, the surviving TH-positive cells were significantly reduced in size compared with the contralateral side (right panel, asterisks, $p<0.001$, paired $t$ test), and there was no difference between the groups, thus indicating that $\mathrm{TH}$-positive neurons rescued by transient GDNF treatment remained in an atrophic state. Error bars represent SEM.

with an orienting movement after the first touch of the wooden probe on either side of the body.

\section{Correlations}

Whereas nigral DA cells were almost completely rescued by GDNF injections over the SN, there was no discernible sparing or regrowth of the TH-positive axon terminals in the striatum. As a consequence, striatal TH-IR fiber density and numbers of surviving TH-IR cells in the SN showed no correlation in the GDNFinjected group ( $\left.p>0.8, r^{2}=0\right)$ (filled circles in Fig. 6), whereas these two parameters were significantly correlated in the vehicleinjected group $(p<0.001),\left(r^{2}=0.7\right)$ (open circles in Fig. 6). As illustrated in the right panel in Figure 6 , the ratio of $\mathrm{TH}$ fiber density to the number of surviving TH-IR cells was reduced by $\sim 80 \%$, from 1.7 in the vehicle-treated group to 0.3 in the GDNFtreated animals.

Striatal TH-IR fiber density of the single experimental groups or of the two groups combined was overall significantly correlated with the animals' performance in both the stepping test $(p<$ $0.001, r^{2}=0.63$ ) (Fig. $7 B$, both groups combined for all values given) and the balance test $\left(p<0.001, r^{2}=0.73\right)$ (Fig. $\left.7 C\right)$. In the stepping test, a residual level of at least $20-25 \%$ of TH-IR fibers in the centrolateral part of the striatum (at the level of the lesion site) was observed as a threshold for initiation of any steps in the forehand direction; thus, all animals displaying a TH-IR fiber density of $<20 \%$ of normal showed a total failure to perform in this test. Performance in the balance test, on the other hand, was close to normal in animals with $\geqslant 30 \%$ of TH-IR fibers in this part of the striatum. Good linear correlation between TH-IR fiber density and balance test scores was seen in the interval between 0 and $30 \%$ of normal TH-IR fiber density. Severe impairment in the balance test score $(<7)$ was seen in animals with maximally $10 \%$ TH-IR fiber-sparing at the level of the lesion site. The scores in the stepping and balance tests were significantly correlated with each other $\left(p<0.01, r^{2}=0.5\right)$. Taken together, these data indicate that forehand stepping and balance test scores, individually or combined, may be used as sensitive measures of striatal DA fiber-sparing or reinnervation in the 6-OHDA lesioned animals.

The animals' performance in the stepping and balance tests was not correlated with nigral cell number when the analysis was performed on all animals, both groups combined, or in the animals in the GDNF-treated group alone. By contrast, the animals in the vehicle-injected group alone displayed significant correlations between stepping or balance test scores and nigral TH-IR cell number ( $p<0.05, r^{2}=0.5 ; p<0.05, r^{2}=0.4$, respectively).

Amphetamine-induced rotational scores (of the single experimental groups or of the two groups combined) showed no significant correlation with any of the other parameters determined. Thus, rotational scores were neither correlated with striatal TH-IR fiber density ( $p>0.05, r^{2}=0.2$ ) (Fig. $7 A$ ) (both groups combined for all values given) or with nigral TH-IR cell number $\left(p>0.1, r^{2}=0\right)$ nor to the performance in the stepping or balance tests ( $p>0.1, r^{2}=0.1 ; p>0.1 ; r^{2}=0.0$, respectively).

\section{DISCUSSION}

This study is the first to report that delayed short-term GDNF treatment can provide long-term rescue of lesioned nigral DA neurons. In the striatal 6-OHDA lesion model, the loss of TH-IR fibers in the striatum has reached its maximum $5 \mathrm{~d}$ after the striatal 6-OHDA injection (Jenkins et al., 1993), and by 1 week, postlesion striatal $\left[{ }^{3} \mathrm{H}\right]$ mazindol-labeled DA uptake sites are maximally reduced (Przedborski et al., 1995). Atrophic changes, but no cell loss, are observed in the SN at this time point (Sauer and Oertel, 1994). The first GDNF injection $5 \mathrm{~d}$ after lesioning, as used here, was thus administered at a time point when the nigral neurons were undergoing degenerative changes, but previous to the onset of cell death, as shown by use of retrograde fluorescent tracer (Sauer and Oertel, 1994).

In previous studies, the neuroprotective effects of GDNF on nigral DA neurons have been determined over shorter postoperative periods, 1-4 weeks after a neurotoxic or surgical lesion (Beck et al., 1995; Kearns and Gash, 1995; Sauer et al., 1995; Tomac et al., 1995). GDNF injected over the SN, either before lesioning or every other day for 1 month starting at the day of lesioning, has been shown to protect DA cells from 6-OHDAinduced cell death as assessed 2-4 weeks later (Kearns and Gash, 1995; Sauer at al., 1995). However, because neurodegeneration in PD progresses over years and decades, during which the nigral DAergic neurons undergo slow degenerative changes (Mc Geer et al., 1988; Fearnley and Lees, 1991; Sawle and Myers, 1993), experimental designs in which neuroprotective factors are supplied after the onset of degeneration should be more relevant for the exploration of their clinical potential.

The present lesion model, therefore, is of particular interest in that it allows study of long-term neuroprotective effects of GDNF in a situation in which the nigral DA neurons are undergoing a sequence of atrophic-degenerative changes that are protracted over several weeks. An additional advantage of this model is that it allows exploration of long-term functional changes in parameters of spontaneous motor behavior of direct relevance for the symptomatology of human PD. 

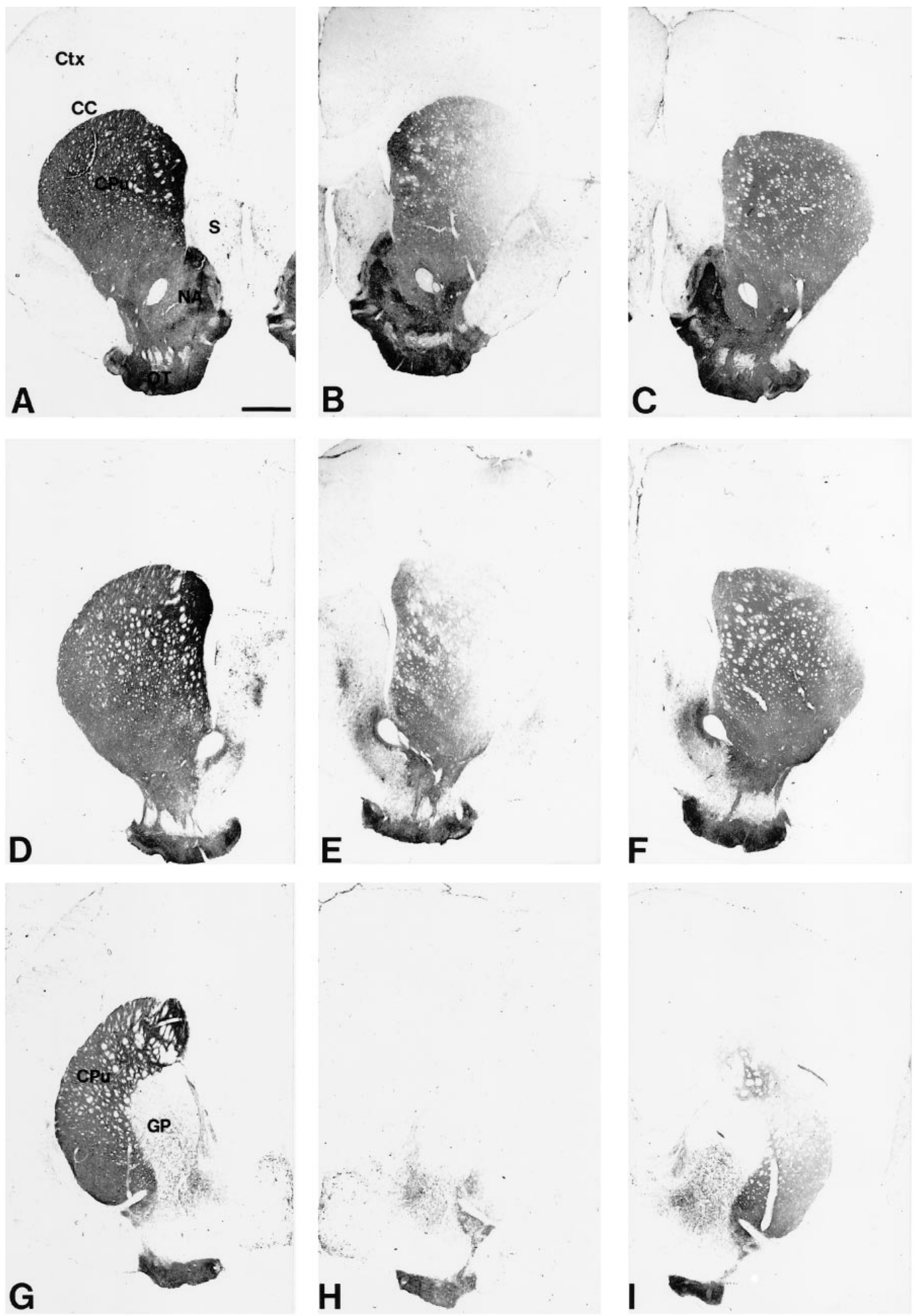

Figure 3. Variability of the striatal 6-OHDA lesion. The lesion-induced loss of striatal TH-IR fiber density varied among the animals, and two extreme cases are illustrated in this figure. $A, D, G$, Three sections illustrating the intact control side (contralateral to the lesion) at the level of the lesion site $(D)$ and rostral $(A)$ or caudal $(G)$ to the lesion site. The levels shown in this figure refer to the measurement of TH-IR fiber density as shown in Figure 4 . $C C$, Corpus callosum; $C P u$, caudate-putamen; $C t x$, cortex; $G P$, globus pallidus; $N A$, nucleus accumbens; $O T$, olfactory tubercle; $S$, septum. $B, E, H$, Corresponding sections from the lesioned striatum of a vehicle-injected animal displaying an extensive loss of TH-IR fiber density in the head and tail of the caudate-putamen. Although the loss of TH-IR is almost complete at the site of the 6-OHDA injection $(E)$ and caudal to it $(H)$, some TH-IR fibers are spared in the medial and the ventrolateral part of the rostral striatum $(B)$. The densitometric analysis of this specimen indicated a $89 \%$ reduction in TH-IR fiber density at the level of the lesion site. $C, F, I$, Lesioned striatum from a vehicle-injected animal displaying a smaller lesion that is largely confined to the dorsolateral part of the caudate-putamen $(F, I)$. Whereas many fibers are spared rostrally from the injection site $(C)$, there is a clear overall reduction of TH-IR fiber density, as seen by the intensity of the staining. The densitometric analysis revealed a $38 \%$ reduction in TH-staining intensity at the level of the lesion site in this animal. Scale bar, $1 \mathrm{~mm}$. 


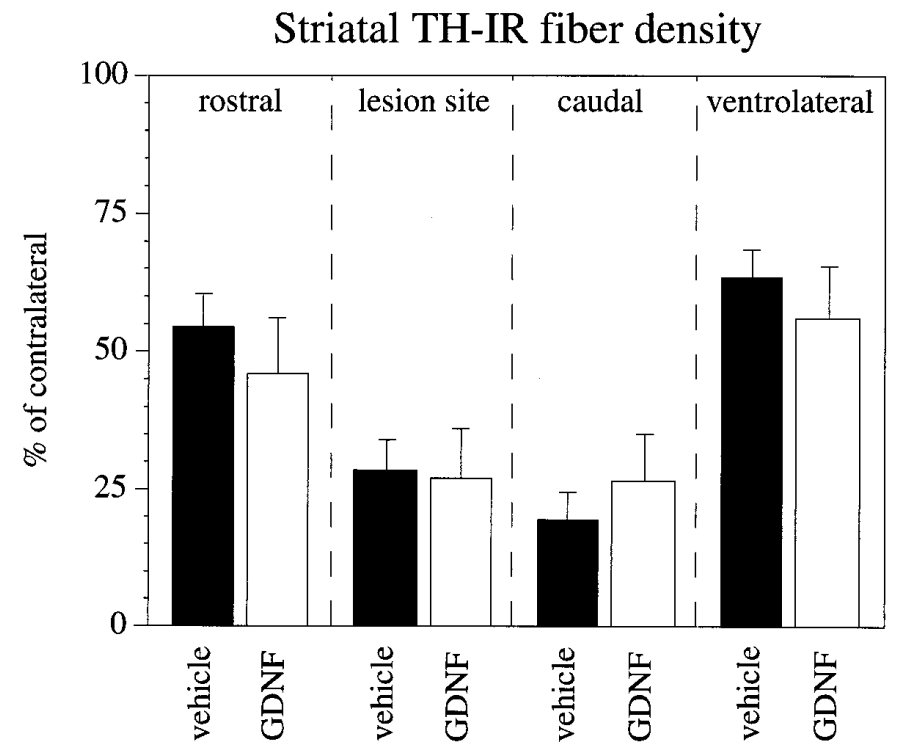

Figure 4. GDNF administration over the SN cell bodies fails to preserve DA nerve terminals in the striatum. Striatal TH-IR fiber density, measured as mean optical density (expressed in percent of the contralateral nonlesioned side) is shown at different rostrocaudal levels in relation to the site of 6-OHDA injection. TH-IR fiber density is significantly different from the contralateral side in both groups at all measured levels $(p<$ 0.001 , paired $t$ test).

\section{Neuroprotection}

The present results show that transient administration of exogenous GDNF adjacent to the cell bodies in the SN for 1 month is sufficient to provide long-lasting survival of the neurotoxindamaged DA neurons and to preserve their local dendritic arborization in the SN pars reticulata. This indicates that GDNF has to be present only during the acute degenerative phase of the retrograde axotomy-induced damage to induce long-lasting, possibly permanent, survival of the affected neurons. However, the persistent $20 \%$ reduction in size of the rescued neurons, which was similar in magnitude to that seen in the vehicle-treated animals, suggests that reversal of the atrophic state may require constant supply of trophic support.

The GDNF treatment used here did not restore DA innervation in the lesioned striatum. In a previous study (Sauer et al., 1995), we observed that GDNF injections over the SN, starting at the day of lesioning, were unable to protect the DA axonal terminals in the striatum against 6-OHDA-induced damage, although the parent cell bodies in the $\mathrm{SN}$ were completely preserved. Interestingly, GDNF treatment starting at a time point (day 5) when the 6-OHDA-induced striatal terminal degeneration is virtually complete, as done here, was not accompanied by any signs of sprouting or regrowth of TH-IR axons in the striatum, showing that application of this neurotrophic factor at the cell body level can afford almost complete protection of the lesioned DA neurons but is insufficient to induce a significant axonal growth response in the treated neurons.

These data are consistent with the observations of Tomac et al. (1995) and Gash et al. (1996) in MPTP-treated mice and monkeys showing that MPTP-induced degeneration of striatal DA terminals was unaffected by injection of GDNF over the SN given 1 week or 3 months after the MPTP treatment. Similar observations have also been made by Sagot et al. (1996) in a model of motoneuron degeneration, in which GDNF was able to reduce the loss of facial motoneurons without having an effect on axon degeneration. Interestingly, however, Tomac et al. (1995) reported significant recovery of striatal DA and striatal TH-positive innervation in animals that had received injection of GDNF directly into the striatum, strongly suggesting that GDNF indeed can induce axonal regrowth or terminal sprouting, provided it is applied close to the lesioned axons. Sprouting of TH-positive fibers around the GDNF injection site also has been reported by Bowenkamp et al. (1995) and Hudson et al. (1995) after single injections of GDNF into the SN.

\section{Lesion-induced behavioral impairments}

A particularly interesting aspect of the present results is the finding that the lesion-induced behavioral impairments were completely unaffected by the GDNF treatment, which demonstrates that preservation of the DA cell bodies and their dendritic projections to the SN pars reticulata is not sufficient for long-lasting functional recovery in this model. Neither long-term deficits in spontaneous motor behavior, studied after the cessation of GDNF treatment, nor amphetamine-induced turning monitored during ongoing GDNF treatment showed any improvement. The latter observation is particularly notable, because previous studies (Hudson et al., 1995) have reported a significant increase in DA turnover as well as an augmentation of the amphetamine-induced response acutely after a single injection of GDNF close to the SN in nonlesioned animals. No similar acute changes in the amphetamine response were observed here.

In the intact nigrostriatal system, DA is known to be released not only from the axon terminals in the striatum but also from the dendrites within the pars reticulata of the $\mathrm{SN}$, where it may play an important role in the modulation of basal ganglia function through the striato-nigral output pathway (Cheramy et al., 1981; Robertson, 1992). Previous pharmacological studies in rodents have shown that the effects of DA-releasing agents (e.g., amphetamine) and DA receptor agonists (e.g., apomorphine) on unconditioned motor behaviors such as rotation and locomotion are mediated in part by activation of DA receptors in the SN (Cheramy et al., 1981; Robertson, 1992), thus raising the possibility that DA released within the nigra itself could compensate for the loss of striatal innervation in the 6-OHDA-lesioned animals. In support of this possibility, Hoffer and collaborators (Hoffer et al., 1994; Bowenkamp et al., 1995) and Nikkhah et al. (1994) have observed reduced apomorphine-induced rotation associated with increased DA levels or TH-positive cells and fibers in the SN after either intranigral GDNF injection or intranigral DA neuron transplants in 6-OHDA-lesioned rats. In the present study, however, neither amphetamine-induced turning nor lesion-induced forelimb akinesia was affected by the GDNF treatment, despite that $\sim 80 \%$ of the nigral DA neurons, as well as their dendritic projections to the SN pars reticulata, was rescued by this treatment. These data underscore the fact that reduction in apomorphineinduced rotation (which reflects the degree of DA-receptor supersensitivity in unilaterally lesioned animals) is not a good predictor of behavioral recovery in either spontaneous motor behavior or in amphetamine-induced turning. Indeed, in the study of intranigral DA neuron transplants by Nikkhah et al. (1994), the reduction in DA-receptor agonist rotation (induced by either apomorphine or by selective D1- and D2-receptor agonists) was not accompanied by any significant improvement in either amphetamine-induced turning or spontaneous motor behaviors.

These data indicate that long-lasting recovery of function by GDNF in the 6-OHDA lesion model is critically dependent on the 


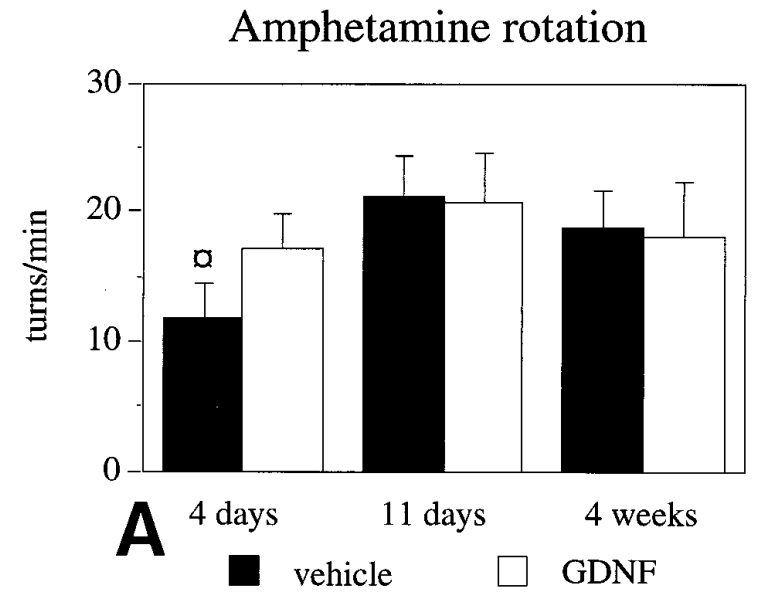

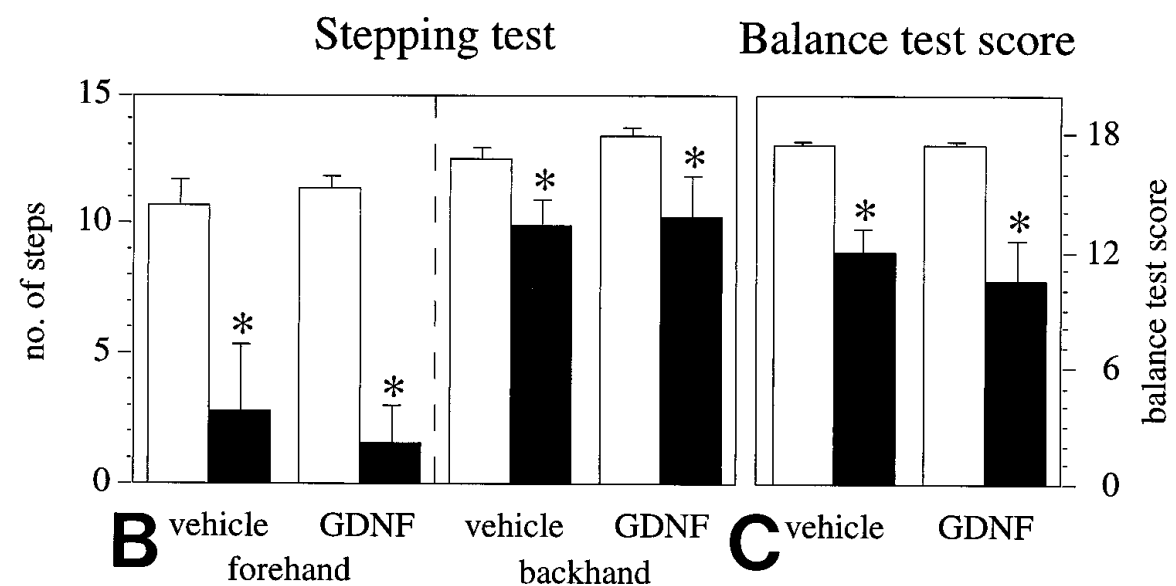

ipsilateral paw contralateral paw
Figure 5. Performance in motor initiation and akinesia tests is impaired in the 6-OHDAlesioned animals. $A$, Ipsilateral rotational net asymmetry scores induced by amphetamine (5 $\mathrm{mg} / \mathrm{kg}$, i.p.), expressed as full-body turns per minute over $90 \mathrm{~min}$. Time points given indicate days after the intrastriatal 6-OHDA lesioning. The high rotational scores seen at $4 \mathrm{~d}$ after lesioning, i.e., the day before the first vehicle or GDNF injection, indicate extensive damage of the striatal DA nerve terminals at the time when GDNF treatment was initiated $(\infty p<0.05$, ANOVA with post hoc Scheffé test). Initiation of forelimb side-stepping movements $(B)$ and the ability of the rats to initiate forelimb movements to regain balance $(C)$ are impaired on the side contralateral to the striatal 6-OHDA lesion. GDNF treatment has no effect on the performance in these tasks. The numbers given are the means of six tests, performed at 4 months after the 6-OHDA lesioning. Asterisks, Significant difference between the paws, paired $t$ test. Error bars represent SEM. reinnervation and, hence, restoration of DA neurotransmission in the denervated striatum. In the present study, the performance of the animals in the forelimb akinesia tests was highly correlated with the extent of TH-positive denervation in the lesioned stria- tum, thus demonstrating that these tests provide highly sensitive measures of the extent of denervation above all in the centrolateral part of the caudate-putamen. This subregion, which was most severely affected by the 6-OHDA lesion coordinates used here,

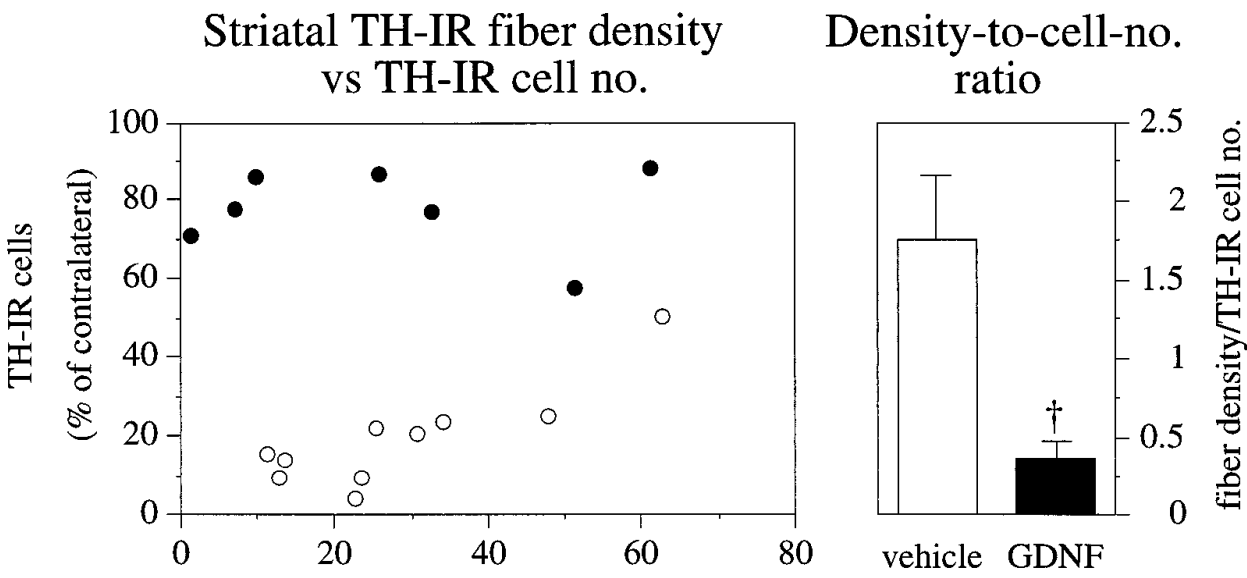

fiber density (\% of contralateral)

Figure 6. Correlation between striatal TH-IR fiber density and TH-IR nigral cell number. In the vehicle-injected animals, the striatal TH-IR fiber density (recorded at the level of the lesion site) is significantly correlated to the number of surviving TH-IR cells in the SN $\left(p<0.001 ; r^{2}=0.7\right.$; open circles), whereas there is no correlation in GDNF-injected animals $(p>0.8$; $r^{2}=0$, filled circles). Similar correlations also were obtained for TH-IR fiber densities recorded rostral or caudal to the lesion site. As nigral cell bodies but not striatal nerve terminals are rescued by supranigral GDNF treatment, the ratio between TH-IR fiber density and TH-IR cell number is drastically reduced. Dagger, Significant difference from the vehicle-injected group ( $p<0.001$, ANOVA with post hoc Scheffé test). Error bars represent SEM. 


\section{Amphetamine rotation vs. striatal TH-IR fiber density}

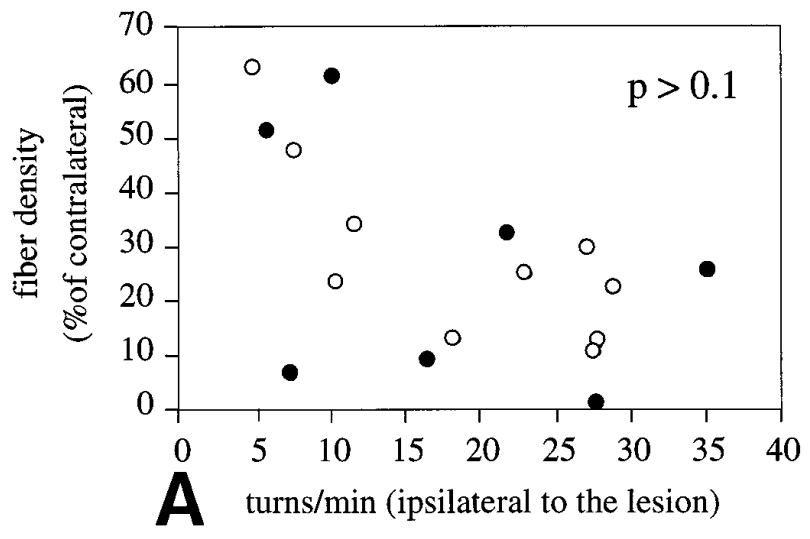

\section{Stepping vs. striatal TH-IR fiber density}

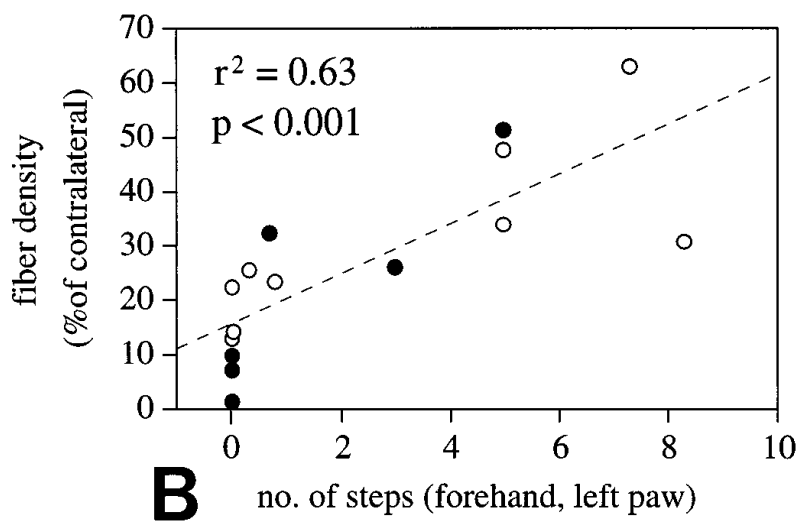

Balance test score vs. striatal TH-IR fiber density

Figure 7. Performance in motor initiation tasks but not in amphetamine-induced rotation is correlated with striatal TH-IR fiber density. $A$, Amphetamine-induced rotation at 4 weeks after lesioning of the single groups or of both groups pooled together shows no correlation with striatal TH-IR fiber density $(p>0.05$, $r^{2}=0.2$, both groups combined). Performance of the animals in the stepping test $(B)$ and the balance test $(C)$ is significantly correlated with striatal TH-IR fiber density (both for the single experimental groups and for the two groups combined), as recorded either at the level of the injection site (shown here) or at the levels rostral and caudal to the injection site (data not shown).

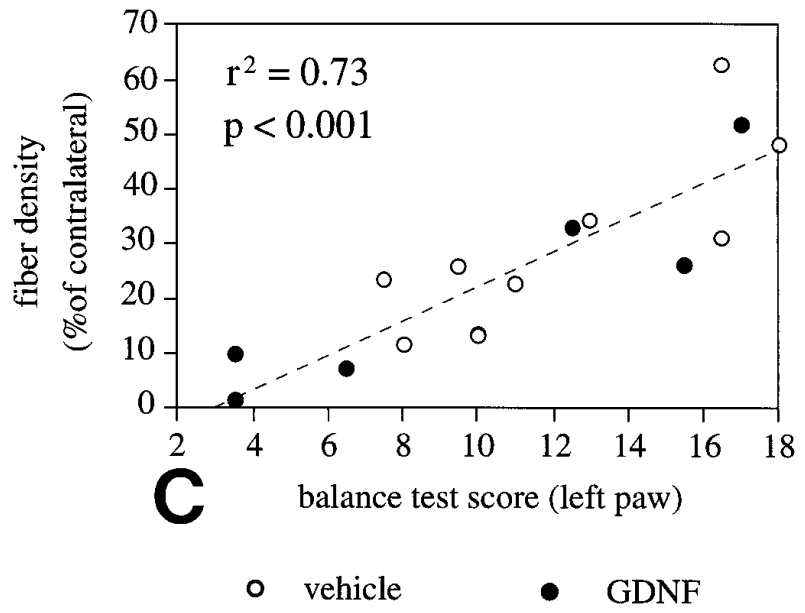

may approximately correspond to the putamen in primates and is therefore likely to be of particular importance in the control of forelimb stepping movements in the rat. By contrast, sensorimotor orientation and disengage behavior were completely unaffected by the lesion, which is consistent with the fact that the TH-positive innervation of the ventrolateral part of the striatum, which is

critically involved in the control of motor initiation behaviors (Dunnett et al., 1983; Mandel et al., 1990), was reduced by only 35-45\%. In the forelimb akinesia tests, marked impairments were seen in animals with a reduction of the TH-positive innervation in the centrolateral part of the striatum of at least $70-75 \%$. Interestingly, the rate of amphetamine-induced rota- 
tion was poorly correlated with the extent of striatal denervation, which indicates that this behavior is less useful as a predictor of regeneration-dependent functional recovery in the present model.

\section{Conclusions}

The present results demonstrate that short-term administration of GDNF at the level of the cell bodies in the SN, starting at a time point when the 6-OHDA-induced axonal degeneration is complete, is sufficient to provide near-complete rescue and long-lasting survival of the lesioned nigral DA neurons. The GDNF-induced sparing of the axotomized nigral DA neurons, however, was not accompanied by any signs of striatal reinnervation or recovery in drug-induced or spontaneous motor behaviors, indicating that functional recovery in the lesioned nigrostriatal DA system requires not only preservation of the nigral cell bodies but also significant reinnervation of the denervated striatal target. Based on parallel studies showing that GDNF may have neurite growth-stimulating properties when applied directly to lesioned or outgrowing axons, we propose that complete recovery from lesion-induced axotomy may be possible to achieve by combined administration of the factor to both the SN and the striatum. Studies designed to test this hypothesis are now under way.

\section{REFERENCES}

Beck KD, Valverde J, Alexi T, Poulsen K, Moffat B, Vandlen RA, Rosenthal A, Hefti F (1995) Mesencephalic dopaminergic neurons protected by GDNF from axotomy-induced degeneration in the adult brain. Nature 373:339-341.

Bowenkamp KE, Hoffman AF, Gerhardt GA, Henry MA, Biddle PT, Hoffer BJ, Granholm AE (1995) Glial cell line-derived neurotrophic factor supports survival of injured midbrain dopaminergic neurons. J Comp Neurol 355:479-489.

Cheramy A, Leviel V, Glowinski J (1981) Dendritic release of dopamine in the substantia nigra. Nature 289:537-542.

Dunnett SB, Björklund A, Schmidt RH, Stenevi U, Iversen SD (1983) Intracerebral grafting of neuronal cell suspensions. IV. Behavioural recovery in rats with unilateral 6-OHDA lesions following implantation of nigral cell suspensions in different forebrain sites. Acta Physiol Scand 522:29-37.

Fearnley JM, Lees AJ (1991) Ageing and Parkinson's disease: substantia nigra regional specificity. Brain 114:2283-2301.

Gash DM, Zhang Z, Ovadia A, Cass WA, Yi A, Simmerman L, Russell D, Martin D, Lapchak PA, Collins F, Hoffer BJ, Gerhardt GA (1996) Functional recovery in parkinsonian monkeys treated with GDNF. Nature 380:252-255.

Hoffer BJ, Hoffman A, Bowenkamp K, Huettl P, Hudson J, Martin D, Lin LF, Gerhardt GA (1994) Glial cell line-derived neurotrophic factor reverses toxin-induced injury to midbrain dopaminergic neurons in vivo. Neurosci Lett 182:107-111.

Hudson J, Granholm AC, Gerhardt GA, Henry MA, Hoffman A, Biddle P, Leela NS, Mackerlova L, Lile JD, Collins F, Hoffer BJ (1995) Glial cell line-derived neurotrophic factor augments midbrain dopaminergic circuits in vivo. Brain Res Bull 36:425-432.
Jenkins R, O'Shea R, Thomas KL, Hunt SP (1993) C-jun expression in substantia nigra neurons following striatal 6-hydroxydopamine neurons in the rat. Neuroscience 53:447-455.

Kearns CM, Gash DM (1995) GDNF protects nigral dopamine neurons against 6-hydroxydopamine in vivo. Brain Res 672:104-111.

Lee CS, Sauer H, Björklund A (1996) Dopaminergic neuronal degeneration and motor impairments following axon terminal lesion by intrastriatal 6-hydroxydopamine in the rat. Neuroscience 72:641-653.

Lin LF, Doherty D, Lile J, Bektesh S, Collins F (1993) A glial cell line-derived neurotrophic factor for midbrain dopaminergic neurons. Science 260:1130-1132.

Mandel RJ, Brundin P, Björklund A (1990) The importance of graft placement and task complexity for transplant-induced recovery of simple and complex sensorimotor deficits in dopamine denervated rats. Eur J Neurosci 2:888-894.

McGeer PL, Itagaki S, Akiyama H, Mc Geer EG (1988) Rate of cell death in parkinsonism indicates active neuropathological process. Ann Neurol 24:574-576.

Nikkhah G, Bentlage C, Cunningham MG, Björklund A (1994) Intranigral fetal dopamine grafts induce behavioral compensation in the rat Parkinson model. J Neurosci 14:3449-3461.

Olsson M, Nikkhah G, Bentlage C, Björklund A (1995) Forelimb akinesia in the rat Parkinson model: differential effects of dopamine agonists and nigral transplants as assessed by a new stepping test. J Neurosci 15:3863-3875.

Paxinos G, Watson C (1986) The rat brain in stereotaxic coordinates, 2nd Ed. San Diego: Academic.

Przedborski S, Levivier M, Jiang H, Ferreira M, Jackson-Lewis V, Donaldson D, Togasaki DM (1995) Dose-dependent lesions of the dopaminergic nigrostriatal pathway induced by intrastriatal injection of 6-hydroxydopamine. Neuroscience 67:631-647.

Riederer P, Wuketich S (1976) Time course of nigrostriatal degeneration in Parkinson's disease. J Neural Transm Park Dis Dement Sect 38:277-301.

Robertson HA (1992) Dopamine receptor interactions: some implications for the treatment of Parkinson's disease. Trends Neurosci 15:201-206.

Sagot Y, Tan SA, Hammang JP, Aebischer P, Kato AC (1996) GDNF slows loss of motoneurons but not axonal degeneration or premature death of pmn/pmn mice. J Neurosci 16:2335-2341.

Sauer H, Oertel WH (1994) Progressive degeneration of nigrostriatal neurons following intrastriatal terminal lesions with 6-OHDA: a combined retrograde tracing and immunocytochemical study in the rat. Neuroscience 59:401-415.

Sauer H, Rosenblad C, Björklund A (1995) GDNF but not TGF- $\beta 3$ prevents delayed degeneration of nigral dopaminergic neurons following striatal 6-hydroxydopamine-lesion. Proc Natl Acad Sci USA 92:8935-8939.

Sawle GV, Myers R (1993) The role of positron emission tomography in the assessment of human neurotransplantation. Trends Neurosci 16:172-176.

Schallert T, Hall S (1988) Disengage sensorimotor deficit following apparent recovery from unilateral dopamine depletion. Behav Brain Res 30:15-24.

Tomac A, Lindquist E, Lin LF, Ögren SO, Young D, Hoffer BJ, Olson L (1995) Protection and repair of the nigrostriatal dopaminergic system by GDNF in vivo. Nature 373:335-339.

Ungerstedt U, Arbuthnott G (1970) Quantitative recording of rotational behavior in rats after 6-hydroxydopamine lesions of the nigrostriatal dopamine system. Brain Res 24:485-493. 